\title{
An efficient importance sampling approach for reliability analysis of time-variant structures subject to time-dependent stochastic load
}

\author{
Xiukai Yuan ${ }^{a, d, *}$, Shaolong $\mathrm{Liu}^{a}$, Matthias Faes ${ }^{b, d}$, Marcos.A. Valdebenito ${ }^{c}$, Michael Beer ${ }^{d, e, f}$ \\ ${ }^{a}$ School of Aerospace and Engineering, Xiamen University, Xiamen 361005, P.R. China \\ ${ }^{b}$ KU Leuven, Department of Mechanical Engineering, LMSD Division, Jan De Nayerlaan 5, St.-Katelijne-Waver, \\ Belgium \\ ${ }^{c}$ Faculty of Engineering and Sciences, Universidad Adolfo Ibáñez, Av. Padre Hurtado 750, 2562340 Viña del \\ Mar, Chile \\ ${ }^{d}$ Institute for Risk and Reliability, Leibniz Universität Hannover, Callinstr. 34, Hannover, Germany \\ ${ }^{e}$ Institute for Risk and Uncertainty, University of Liverpool, Peach Street, L69 7ZF Liverpool, United Kingdom \\ ${ }^{f}$ International Joint Research Center for Engineering Reliability and Stochastic Mechanics, Tongji University, \\ Shanghai 200092, China
}

\begin{abstract}
Structural performance is affected by deterioration processes and external loads. Both effects may change over time, posing a challenge for conducting reliability analysis. In such context, this contribution aims at assessing the reliability of structures where some of its parameters are modeled as random variables, possibly including deterioration processes, and which are subjected to stochastic load processes. The approach is developed within the framework of importance sampling and it is based on the concept of composite limit states, where the time-dependent reliability problem is transformed into a series system with multiple performance functions. Then, an efficient two-step importance sampling density function is proposed, which splits time-invariant parameters (random variables) from the time-variant ones (stochastic processes). This importance sampling scheme is geared towards a particular class of problems, where the performance of the structural system exhibits a linear dependency with respect to the stochastic load for fixed time. This allows calculating the reliability associated with the series system most efficiently. Practical examples illustrate the performance of the proposed approach.
\end{abstract}

Keywords: Time-variant structure, Stochastic load, Importance sampling, Composite limit state functions, Simulation-based method, Cumulative failure probability

\section{Introduction}

In the past decades, structural reliability theory for time-invariant problems has been widely 2 investigated and developed. Following this framework, it is assumed that the system and its 3 
characteristics are static, and random variables are used to characterize their natural variabil- 4 ity. Various methods have been developed to carry out static reliability analysis, which can be 5 broadly classified as: asymptotic analytical methods, such as first/second order reliability method 6 (FORM/SORM) [1]; and simulation-based methods, e.g., Monte Carlo simulation (MCS)[2][3], 7 importance sampling (IS)[4, 5], line sampling [6] and subset simulation [7]. The efficiency of both 8 classes of methods can be improved by applying surrogate methods such as response surface meth- 9 ods [8], Kriging [9, 10] support vector machines [11] and polynomial chaos expansion [12], among 10 others.

Although advances in time-invariant reliability problems have been far reaching, in realistic 12 engineering situations, the model parameters typically change as a function of time, which is 13 termed as time-dependent (or time-variant). This is a result of the fact that engineering structures 14 and systems are often exposed to severe operating or environmental conditions during their service $\quad 15$ life, which are responsible for the deterioration of structural strength and stiffness with time [13]. 16 Furthermore, the intensity and frequency of loads acting on these systems may also vary with 17 time. A reliability analysis can properly reflect and quantify the effect of time-variant factors 18 by estimating the failure probability of a system/structure over a period of time. Because time 19 is considered, more challenges are faced as compared with traditional, time-invariant reliability 20 analysis. As such, the application of typically applied reliability engineering methods may not be ${ }^{21}$ direct in this context.

Reliability analysis considering time-variant properties and loadings has attracted much at- ${ }_{23}$ tention recently and a vast number of methods have been developed. These are roughly classified 24 into three categories: (1) the out-crossing rate based methods; (2) the extreme value methods; 25 and (3) the composite limit state methods. Methods based on out-crossing rate make use of the ${ }^{26}$ relationship between the failure probability and the expected mean number of out-crossings of ${ }_{27}$ the random process over a prescribed threshold. There are many different approximations to the 28 out-crossing rate available in the literature, see e.g. $[14,15,16]$. However, the main drawback of 29 methods based on out-crossing rate for reliability analysis is that they are based on the assump- 30 tion of independence and Poisson distribution, which in certain cases may lead to a low accuracy. $\quad 31$ Methods based on extreme values consider the worst situation of system's performance over the 32 time interval of interest, and whenever the extreme value of the limit state function exceeds a 33 given threshold, failure occurs. The key challenge in extreme value methods lies in the construc- 34 
tion of a proper surrogate model or a probability distribution for the output random process that 35 characterizes the structural performance [17]. In this context, a Gaussian process (GP) model 36 has been used in [18] to represent the extreme system response over time. Later, Hu and Ma- 37 hadevan [19] proposed a single-loop GP approach where training points of random variables over 38 time are generated at once (instead of tracking time and maximum responses separately). Qian 39 et al. [20] also proposed a single-loop strategy for time-variant system reliability analysis by com- 40 bining multiple response Gaussian process models. Many surrogate methods are only applicable ${ }_{41}$ to cases where no input random process are involved. In [21] and [22], surrogate model methods 42 have been proposed to address this issue. However, discrete representation of stochastic processes 43 increases the dimensionality of the problem, posing a challenge for surrogate modeling due to the $\quad 44$ so-called curse of dimensionality. An alternative strategy to surrogate modelling schemes is to 45 fit a probability distribution for the extreme values by a suitable distribution estimation method. $\quad 46$ $\mathrm{Hu}$ and $\mathrm{Du}[17]$ proposed in this context a method for constructing an extreme value distribution, $\quad 47$ based on the expansion optimal linear estimation method (EOLE) and saddle-point approxima- 48 tion. However, the distribution of extreme values in some cases may be highly non-linear and/or 49 follow a multimodal distribution, posing additional challenges. A third group of methods is based 50 on the concept of a composite limit state function, which serves as an alternative to handle re- 51 liability problems with time-variant characteristics. The main idea behind composite limit state 52 methods is that the original time-variant limit state function is discretized into a sequence of 53 instantaneous ones, and then the concept of series system reliability is used to convert the time- $\quad 54$ variant reliability analysis into a time-invariant one. Jiang et al. [23] used time discretization 55 to convert stochastic processes into random variables, and then the first-order reliability method 56 (FORM) is adopted to compute the probability associated with the linearized limit-state function. $\quad 57$ Mourelatos et al. [24], based on the concept of composite limit state, used the total probability 58 theorem and FORM to calculate reliability of time-dependent problems. Also, this composite 59 limit state idea allows applying simulation-based methods for static system reliability analysis in 60 time-variant problems. Recently, Li at al. [25] proposed a Generalized subset simulation (GSS) to 61 handle high-dimensional time-variant reliability. Similarly, Du et al. [26] adopted parallel subset ${ }_{62}$ simulation to handle time-variant reliability with both deterioration in material properties and 63 dynamic load.

Several of the methods for reliability analysis which have been developed so far consider load as $\quad 65$ 
stochastic excitation which is dependent on time, while parameters related with structural behavior $\quad 66$ are represented either as (static) random variables or even deterministic. For example, Au and 67 Beck [27] proposed an efficient importance sampling method for linear systems; later, Misraji et al. $\quad 68$ [28] applied a directional importance sampling scheme for reliability analysis of structural systems 69 subject to stochastic Gaussian loading. When uncertainties on different types of parameters are 70 simultaneously considered, i.e., random structural variables, time-variant structural parameters 71 (due to deterioration, etc.) and stochastic load processes, the reliability problem comprises a 72 time-variant structure (system), which is subjected to time-variant loads. In this context, most 73 of the current methods for reliability are based on approximate analytical methods, i.e., FORM 74 [23][24], or resort to surrogate models, i.e., through building extreme value surrogate models, 75 as in $[29,18,19,20,21,22]$, which have their own potential shortcomings. In the context of 76 simulation-based methods, MCS and subset simulation can be used to solve reliability problems 77 involving time-variant structures subject to stochastic load. However, their practical application 78 may become unfeasible due to the prohibitively high computational associated with uncertainty 79 propagation. Hence, there is still a large space for developing simulation-based methods for solving 80 this kind of reliability problems in an efficient manner. 81

In view of the aforementioned difficulty in estimating the reliability of time-variant struc- $\quad 82$ tures subject to time-dependent loads, this contribution focuses on a specific type of problems, 83 namely conditional linear time-variant systems. By time-variant, it is meant that the time-variant 84 structure (with time-variant structural properties) is subjected to time-variant loads (stochastic 85 process); and by conditional linear, it is meant that at a particular instant of time and for a 86 nominal structural parameter setting, the output response has a linear relationship with the load. $\quad 87$ The proposed approach is developed within the context of the composite limit state concept and 88 transforms the time-variant problem into a series systems reliability problem. As linear time- 89 variant systems are considered, an extremely efficient importance sampling density (ISD) function 90 is proposed to compute the reliability of the transformed series system. The importance sampling 91 scheme splits and explores the stochastic space spanned by the static random variables and the 92 time-variant space spanned by the stochastic processes in two steps. First, samples are generated 93 in the space associated with static parameters, after which conditional samples are generated 94 according to a specially designed ISD in the time-variant space. This allows to compute the re- 95 liability associated with the transformed series system efficiently. The innovative aspects of this 96 
study with respect to the state-of-the-art are as follows:

- A new tool for structural time-variant reliability analysis is presented, in which input random 98 variables, structural degradation processes as well as stochastic excitation processes are 99 included. 100

- A simulation-based method which can produce satisfactory, accurate estimations of the 101 failure probability is formulated. 102

- The most salient feature of the proposed approach is that it entails a single straightforward 103 simulation scheme, where neither optimization is applied to find the extreme response values 104 over time nor approximate linearization with respect to parameters is required. 105

This contribution is organized as follows. In Section 2, the definition and the transformation of 106 time-dependent reliability problem is discussed. Then, the mathematical formulation of the pro- ${ }_{107}$ posed framework is developed in Section 3. Section 4 illustrates the performance of the proposed 108 approach through a number of application examples. Section 5 closes the paper with discussions 109 and an outlook for future work.

\section{Reliability of structures with time-variant behavior}

\subsection{Reliability definition}

In general, reliability problems whose performance is time-variant can be classified into three 113 groups according to the coupling and nature of the uncertain parameters [17]: (1) $G=g(\boldsymbol{x}, t) \quad 114$ where $G$ is a response of interest, which is determined by the limit state function $g(\cdot)$; $t$ is the 115 time instant; $\boldsymbol{x}=\left[x_{1}, x_{2}, \ldots, x_{n}\right]$ is the vector of basic time-invariant random variables of the 116 structure/system with probability density function $f(\boldsymbol{x}) ;(2) G=g(\boldsymbol{x}, \boldsymbol{Y}(t))$ where $\boldsymbol{Y}(t)$ are ${ }_{117}$ the time-dependent stochastic processes, which are implicit with respect to time $t ;(3) G={ }_{118}$ $g(\boldsymbol{x}, t, \boldsymbol{Y}(t))$ which is the most general type of problem with time-invariant random variables, 119 explicit function terms with respect to time (such as structural degradation processes), and time- ${ }_{120}$ dependent stochastic processes (such as stochastic load processes). ${ }^{121}$

In this contribution, the last, most general type of problems is considered. The corresponding 122 cumulative failure probability over the time period is given by:

$$
P_{f}(0, T)=P\{G=g(\boldsymbol{x}, t, \boldsymbol{Y}(t)) \leq 0, \exists t \in[0, T]\}
$$


where $[0, T]$ is the time interval of interest; $\exists$ stands for 'there exists at least one'; $\boldsymbol{Y}(t)={ }^{124}$ $\left[Y_{1}(t), \ldots, Y_{n_{Y}}(t)\right]$ is the vector of time-dependent stochastic processes with respect to time $t, \quad 125$ which refers to load; $g(\cdot)$ is the corresponding limit state function. In this contribution, the focus 126 is on conditional linear time-variant structural systems. That is, the response of interest is linear ${ }_{127}$ with respect to the input load conditional on a given time instant and structural parameters fixed 128 at certain values; from a mathematical viewpoint, the load terms appears in the form of a linear ${ }_{129}$ term in the limit state function, which can be given by (in case it is explicitly available): 130

$$
g(\boldsymbol{x}, t, \boldsymbol{Y}(t))=a(\boldsymbol{x}, t)+\sum_{i=1}^{n_{Y}} b_{i}(\boldsymbol{x}, t) Y_{i}(t)
$$

where $a(\boldsymbol{x}, t)$ and $b_{i}(\boldsymbol{x}, t)$ can be any type of function(implicit or explicit) of structural random 131 variables and time, and $Y_{i}(t)$ appears as a linear term. Note that even if the limit state function 132 cannot be stated explicitly, in case that a linear relationship between the response and load at 133 fixed time is verified, the proposed approach can also be applied. Such is the case, for example, in 134 linear structural analysis, where the response (deformation, stress and strain, etc.) is linear with 135 respect to the load.

\subsection{Composite limit states transformation of reliability problem with time-variant behavior}

The composite limit state is a useful approach to handle reliability problems with time- ${ }_{138}$ dependent behavior. Its basic idea is to use the concept of series system reliability of the instanta- $\quad 139$ neous limit state functions to covert the time-dependent reliability problem into a time-invariant 140 one. First, the time interval $[0, T]$ is discretized using a time step size $\triangle t$. Then, a time sequence ${ }_{141}$ $\left[t_{0}, \ldots, t_{l} \ldots, t_{m}\right]=[0, \ldots, l \triangle t, \ldots, m \triangle t]$ is generated, where $l=0, \ldots, m$ is the time index, $t_{0}=0 \quad{ }_{142}$ and $t_{m}=m \triangle t=T$. Based on this time discretization, the instantaneous failure probability is: $\quad{ }_{143}$

$$
\left.P_{f}\left(t_{l}\right)=P\left\{G_{l}=g\left(\boldsymbol{x}, t_{l}, \boldsymbol{Y}\left(t_{l}\right)\right) \leq 0\right)\right\}
$$

where $G_{l}=g\left(x, t_{l}, \boldsymbol{Y}\left(t_{l}\right)\right)$ is the instantaneous limit state function at time instant $t_{l}$.

Based on the series system reliability formulation, the cumulative failure probability at time ${ }_{145}$ instant $T$ is given by:

$$
P_{f}(0, T)=P\left\{\bigcup_{l=0}^{m} F_{l}\right\}=P\left\{\min _{l=0, \ldots, m} g\left(\boldsymbol{x}, t_{l}, \boldsymbol{Y}\left(t_{l}\right)\right) \leq 0\right\}
$$


where $F_{l}=\left\{G_{l} \leq 0\right\}$ is the failure region associated with the limit state function at the $l$-th time $\quad 147$ instant, that is $G_{l}$. The failure probability can be expressed as an integral given by: ${ }_{148}$

$$
\begin{gathered}
P_{f}(0, T)=\iint I_{F}\left[\boldsymbol{x}, t_{0}, \ldots, t_{m}, \boldsymbol{Y}\left(t_{1}\right), \ldots, \boldsymbol{Y}\left(t_{m}\right)\right] f(\boldsymbol{x}) \\
f\left(\boldsymbol{Y}\left(t_{0}\right)\right) \ldots f\left(\boldsymbol{Y}\left(t_{m}\right)\right) \mathrm{d} \boldsymbol{x} \mathrm{d} \boldsymbol{Y}\left(t_{0}\right) \ldots \mathrm{d} \boldsymbol{Y}\left(t_{m}\right)
\end{gathered}
$$

where $F=\bigcup_{l=0}^{m}\left\{F_{l}: G_{l} \leq 0\right\}$ is the failure of the series system; $f(\boldsymbol{x})$ is the probability distribution 149 function of the (static) random variables; and $I_{F}[\cdot]$ is the indicator function, which is equal to 150 1 in case failure occurs at any time instant within $[0, T]$. Note that the computation of this 151 failure probability is far from trivial, as it is a series system problem [25, 30]. Of all simulation- 152 based reliability analysis methods, Monte Carlo simulation is a widely used technique which can 153 be applied to estimate this failure probability. However, its drawback is its low efficiency for 154 estimating small failure probabilities.

\section{Proposed approach}

This section presents an efficient importance sampling reliability analysis approach for systems $\quad 157$ with time-dependent properties. Section 3.1 first describes the spectral decomposition method for $\quad 158$ modeling the input stochastic process associated with the load. The strategy for coping with 159 time-dependent limit state functions by transforming the problem to a series system is discussed 160 in Section 3.2. Section 3.3 presents the proposed importance sampling density function and Section 161 3.4 summarizes the procedure of proposed approach.

\subsection{Spectral decomposition of input random process}

In case an analyst is faced with aleatory uncertainties, the uncertain quantities are usually ${ }_{164}$ modeled as random variables. However, when these quantities vary over time (or space), they 165 should be treated as random processes. Note that there are different kinds of random processes [31]. 166 In the context of reliability analysis, a common approach is to transform them into traditional 167 random variables via an appropriate spectral decomposition such as the Karhunen-Loève (K-L) ${ }_{168}$ expansion [32, 33] or the Expansion Optimal Linear Estimator (EOLE) [34]. 169

Consider an Gaussian, nonstationary stochastic process $Y(t)$ defined by the mean function 170 $\mu_{Y}(t)$, the standard deviation function $\sigma_{Y}(t)$, and the auto-correlation function $\rho_{Y}\left(t_{l}, t_{j}\right)$. The ${ }_{171}$ covariance function of this process between time $t_{l}$ and $t_{j}$ is calculated by:

$$
\operatorname{Cov}\left(t_{l}, t_{j}\right)=\rho_{Y}\left(t_{l}, t_{j}\right) \sigma_{Y}\left(t_{l}\right) \sigma_{Y}\left(t_{j}\right)
$$


When the time interval is discretized as $\left[t_{0}, \ldots, t_{l} \ldots, t_{m}\right]$, the corresponding covariance matrix 173 $\Sigma_{Y}$ is formed as

$$
\boldsymbol{\Sigma}_{Y}=\left[\begin{array}{cccc}
\operatorname{Cov}\left(t_{0}, t_{0}\right) & \operatorname{Cov}\left(t_{0}, t_{1}\right) & \cdots & \operatorname{Cov}\left(t_{0}, t_{m}\right) \\
\operatorname{Cov}\left(t_{1}, t_{0}\right) & \operatorname{Cov}\left(t_{1}, t_{1}\right) & \cdots & \operatorname{Cov}\left(t_{1}, t_{m}\right) \\
\vdots & \vdots & \ddots & \vdots \\
\operatorname{Cov}\left(t_{m}, t_{0}\right) & \operatorname{Cov}\left(t_{m}, t_{1}\right) & \cdots & \operatorname{Cov}\left(t_{m}, t_{m}\right)
\end{array}\right]
$$

In case $\boldsymbol{\Sigma}_{Y}$ is symmetric, bounded and positive-definite, $Y(t)$ can be represented following the 175 K-L expansion as [32]:

$$
Y(t)=\mu_{Y}(t)+\sum_{p=1}^{k} \sqrt{\lambda_{p}} \Psi_{p}(t) Z_{p}
$$

where $\Psi_{p}(t)$ corresponds to the element of matrix $\Psi$ located in the row associated with time 177 $t$ and the $p$-th column; $\Psi=\left[\Psi_{1}, \Psi_{2}, \ldots, \Psi_{k}\right]$ is the matrix of orthogonal eigenvectors and 178 $\boldsymbol{\Lambda}=\operatorname{diag}\left[\lambda_{1}, \lambda_{2}, \ldots, \lambda_{m}\right]$ is a diagonal matrix that contains the corresponding non-negative 179 eigenvalues obtained by performing eigendecomposition $\boldsymbol{\Sigma}_{Y}=\Psi \Lambda \Psi^{T} ; k \leq m$ is the number of ${ }_{180}$ identified dominant eigenfunctions, where $k$ must be selected such that a well-selected error on 181 the reconstructed variance of the process is minimized [35]. Since $Y(t)$ is a Gaussian process, 182 $Z_{p}, p=1, \ldots, k$ are i.i.d. standard Normal random variables. This means that the random 183 process $Y(t)$ can be represented by a number of standard Gaussian random variables, and its 184 standard deviation $\sigma_{Y}(t)$ can be obtained as:

$$
\sigma_{Y}(t)=\sqrt{\sum_{p=1}^{k} \lambda_{p} \Psi_{p}^{2}(t)}
$$

Note that the approach that is introduced in the following section can be applied to various 186 kinds of stochastic process once they are represented by independent normal variables, no matter $\quad 187$ what series expansion methods are used. As an additional remark, it should be noted that the 188 proposed framework is applicable for problems involving Gaussian stochastic processes only. In 189 fact, non Gaussian processes cannot be considered directly within the proposed scheme. $\quad 190$

\subsection{Reliability expressed in terms of a series system}

$$
Y_{i}(t)=\mu_{Y_{i}}(t)+\sum_{p=1}^{k_{i}} \sqrt{\lambda_{i p}} \Psi_{i p}(t) Z_{i p}
$$


where $k_{i}$ is the number of retained eigenfunctions in the K-L expansion, and the corresponding 193 standard deviation can be obtained as:

$$
\sigma_{Y_{i}}(t)=\sqrt{\sum_{p=1}^{k_{i}} \lambda_{i p} \Psi_{i p}^{2}(t)}
$$

Then the instantaneous limit state function in Eq. (2) can be rewritten as:

$$
g_{Z}\left(\boldsymbol{x}, t_{l}, \boldsymbol{Z}\right)=a\left(\boldsymbol{x}, t_{l}\right)+\sum_{i=1}^{n_{Y}} b_{i}\left(\boldsymbol{x}, t_{l}\right) \mu_{Y_{i}}\left(t_{l}\right)+\sum_{i=1}^{n_{Y}} \sum_{p=1}^{k_{i}} b_{i}\left(\boldsymbol{x}, t_{l}\right) \sqrt{\lambda_{i p}} \Psi_{i p}(t) Z_{i p}
$$

where $\mathbf{Z}=\left[Z_{11}, \cdots, Z_{1 k_{1}}, Z_{21}, \cdots, Z_{2 k_{2}}, \cdots, Z_{n_{Y} 1}, \cdots, Z_{n_{Y} k_{n_{Y}}}\right]$ is the vector collecting all Gaus- 196 sian random variables involved.

Considering the limit state function (LSF) given in Eq. (12), the corresponding failure proba- ${ }_{198}$ bility, as defined in Eq. (4), is expressed as:

$$
P_{f}(0, T)=P_{f}\left(0, t_{m}\right)=\iint I_{F_{m}^{U}}\left[\boldsymbol{x}, t_{0}, \cdots, t_{m}, \boldsymbol{Z}\right] \phi(\boldsymbol{Z}) f(\boldsymbol{x}) \mathrm{d} \boldsymbol{x} \mathrm{d} \boldsymbol{Z}
$$

where $F_{m}^{U}=\bigcup_{l=0}^{m}\left\{F_{l}: g_{Z}\left(\boldsymbol{x}, t_{l}, \boldsymbol{Z}\right) \leq 0\right\}$ is the union of failure events of the series system; $I_{F_{m}^{U}}(\cdot){ }_{200}$ is the indicator function of $F_{m}^{U}$; and $\phi(\cdot)$ is the joint PDF of i.i.d. standard Gaussian variables. 201

Note that direct solution of this equation using standard quadrature schemes is not possible 202 due to the typically high number of random variables involved. Therefore, simulation methods are 203 preferred to approximate this integral, which may require a high number of LSF evaluations to 204 obtain an acceptable coefficient of variation [36]. This motivates the use of importance sampling 205 methods [37] to reduce the required number of LSF evaluations, as recently also successfully applied 206 in the context of calculating first passage probabilities in linear dynamical systems [28, 38]. The 207 next section deals with the development of an importance sampling density for this specific case. 208

\subsection{Importance sampling density function}

In this section, an efficient importance sampling density (ISD) function is formulated, which 210 has the following form:

$$
H(\boldsymbol{x}, \boldsymbol{Z})=H(\boldsymbol{Z} \mid \boldsymbol{x}) H(\boldsymbol{x})
$$

Inspection of Eq.(14) reveals that this importance sampling density consists of two parts: one ${ }^{212}$ part related with the (static) random variables $\boldsymbol{x}$ and another part to handle the stochastic process ${ }_{213}$ $Y(t)$ via its associated random variables $\boldsymbol{Z}$. Recall that $\boldsymbol{x}$ is regarded as static because it does not ${ }^{214}$ vary with time. In other words, when a sample of $\boldsymbol{x}$ is generated, it becomes a constant during ${ }_{215}$ the time interval $[0, T]$. 


\subsubsection{Importance sampling density function for static random variables}

The fist step of the proposed framework is to determine $H(\boldsymbol{x})$. The most straightforward way ${ }_{218}$ is to just choose the original distribution as the ISD, which is :

$$
H(\boldsymbol{x})=f(\boldsymbol{x})
$$

Undoubtedly, $H(\boldsymbol{x})$ may also be constructed based on information from the failure region, consid- ${ }^{220}$ ering design points or an adaptive importance sampling density function. Nonetheless, it may be 221 challenging to determine a proper importance sampling function associated with the series system 222 in Eq. (2). Besides, extra computational burden is involved [30]. It is also worthy to note that ${ }^{223}$ sampling of $H(\boldsymbol{x})$ can be done applying low-discrepancy sequences [5], which are especially suit- ${ }^{224}$ able for cases where a small number of samples is used. It will be shown in examples section that ${ }_{225}$ the proposed approach just needs hundreds of samples, and it expected to achieve an improved 226 convergence rate in case the low-discrepancy sequence is adopted.

\subsubsection{Importance sampling density function for $\boldsymbol{Z}$}

The next step is to formulate the probability function associated with $\boldsymbol{Z}$ conditioned on $\boldsymbol{x}$. ${ }^{229}$ Once the ISD of $H(\boldsymbol{x})$ is determined, a number of samples $\left\{\boldsymbol{x}^{(j)}:(j=1, \ldots, N)\right\}$ can be generated ${ }_{230}$ according to $H(\boldsymbol{x})$. Hence, in the following, it is considered that $\boldsymbol{x}$ has assumed a fixed value. 231 Then, $H(\boldsymbol{Z} \mid \boldsymbol{x})$ can be determined based on this fixed value.

Recall the transformed instantaneous $\operatorname{LSF} g_{Z}\left(\boldsymbol{x}, t_{l}, \boldsymbol{Z}\right)$ of Eq. (12). It is readily noticed that ${ }^{233}$ for a fixed value $\boldsymbol{x}$, the LSF is only an expression depending on $\boldsymbol{Z}$. As $\boldsymbol{Z}$ is a vector of i.i.d. 234 normal variables, the corresponding mean and standard deviation of LSF (conditioned on $\boldsymbol{x}$ ) can 235 be easily obtained:

$$
\begin{gathered}
\mu_{g_{Z}}^{l}(\boldsymbol{x})=\mu_{g_{Z}}\left(\boldsymbol{x}, t_{l}\right)=a\left(\boldsymbol{x}, t_{l}\right)+\sum_{i=1}^{n_{Y}} b_{i}\left(\boldsymbol{x}, t_{l}\right) \mu_{Y_{i}}\left(t_{l}\right) \\
\sigma_{g_{Z}}^{l}(\boldsymbol{x})=\sigma_{g_{Z}}\left(\boldsymbol{x}, t_{l}\right)=\sqrt{\sum_{i=1}^{n_{Y}} \sum_{p=1}^{k_{i}} b_{i}^{2}\left(\boldsymbol{x}, t_{l}\right) \lambda_{i p} \Psi_{i p}^{2}(t)}=\sqrt{\sum_{i=1}^{n_{Y}} b_{i}^{2}\left(\boldsymbol{x}, t_{l}\right) \sigma_{Y_{i}}^{2}\left(t_{l}\right)}
\end{gathered}
$$

The reliability index for the instantaneous limit state function at time instant $t_{l}$ in Eq. (12) ${ }_{237}$ can be obtained as:

$$
\beta\left(\boldsymbol{x}, t_{l}\right)=\frac{\mu_{g_{Z}}^{l}(\boldsymbol{x})}{\sigma_{g_{Z}}^{l}(\boldsymbol{x})}
$$


and the corresponding design point $\boldsymbol{Z}_{l}^{*}(\boldsymbol{x})=\left[Z_{l, 1}^{*}(\boldsymbol{x}), \cdots, Z_{l, p}^{*}(\boldsymbol{x}), \cdots, Z_{l, n_{Z}}^{*}(\boldsymbol{x})\right]$ can be obtained ${ }^{239}$ as:

$$
Z_{l, p}^{*}(\boldsymbol{x})=-\frac{b_{i}\left(\boldsymbol{x}, t_{l}\right) \sqrt{\lambda_{i p}} \Psi_{i p}(t) \mu_{g_{Z}}^{l}(\boldsymbol{x})}{\sum_{i=1}^{n_{Y}} b_{i}^{2}\left(\boldsymbol{x}, t_{l}\right) \sigma_{Y_{i}}^{2}\left(t_{l}\right)}
$$

The distribution of the random variables $(\boldsymbol{Z})$ conditional on the failure region $F_{l}(\boldsymbol{x})$ (which is ${ }^{241}$ defined as $\left.F_{l}(\boldsymbol{x})=\left\{g_{Z}\left(\boldsymbol{x}, t_{l}, \boldsymbol{Z}\right) \leq 0\right\}\right)$ is given by [27]:

$$
f\left(\boldsymbol{Z} \mid F_{l}(\boldsymbol{x})\right)=\frac{\phi(\boldsymbol{Z}) I_{F_{l}}(\boldsymbol{x}, \boldsymbol{Z})}{\Phi\left[-\beta\left(\boldsymbol{x}, t_{l}\right)\right]}
$$

where $I_{F_{l}}(\cdot)$ is the indicator function of $F_{l}(\boldsymbol{x}) ; \Phi(\cdot)$ is the cumulative distribution function $(\mathrm{CDF}) \quad{ }_{243}$ of the standard Gaussian variable. An expression for generating samples of $\boldsymbol{Z}$ conditional on $F_{l}(\boldsymbol{x}) \quad{ }_{244}$ is given by [27] :

$$
\boldsymbol{Z}=\alpha \boldsymbol{e}_{l}^{*}(\boldsymbol{x})+\boldsymbol{U}^{\perp}
$$

where $\alpha$ is a standard Gaussian random variable conditional on $\alpha \geq \beta\left(\boldsymbol{x}, t_{l}\right) ; \boldsymbol{e}_{l}^{*}(\boldsymbol{x})$ is a unit vector ${ }_{246}$ in the direction of the design point which is given by:

$$
\boldsymbol{e}_{l}^{*}(\boldsymbol{x})=\frac{\boldsymbol{Z}_{l}^{*}(\boldsymbol{x})}{\left\|\boldsymbol{Z}_{l}^{*}(\boldsymbol{x})\right\|}=\frac{\boldsymbol{Z}_{l}^{*}(\boldsymbol{x})}{\beta\left(\boldsymbol{x}, t_{l}\right)}
$$

and $\boldsymbol{U}^{\perp}$ is a standard Gaussian random vector orthogonal to $\boldsymbol{e}_{l}^{*}(\boldsymbol{x})$, which is described by [27]: ${ }_{248}$

$$
\boldsymbol{U}^{\perp}=\boldsymbol{U}-\left\langle\boldsymbol{U}, \boldsymbol{e}_{l}^{*}(\boldsymbol{x})\right\rangle \boldsymbol{e}_{l}^{*}(\boldsymbol{x})
$$

with $\langle\cdot, \cdot\rangle$ denoting the inner product operator; and $\boldsymbol{U}$ is a $n$-dimensional standard Gaussian vector. $\quad 249$ Note that $n$ represents the total number of random variables associated with the representation 250 of the stochastic processes, that is $n=\sum_{i=1}^{n_{Y}} k_{i}$. Thus, the final expression for generating samples ${ }_{251}$ of $\boldsymbol{Z}$ which are distributed as $f\left(\boldsymbol{Z} \mid F_{l}(\boldsymbol{x})\right)$ is:

$$
\boldsymbol{Z}=\boldsymbol{U}+\left(\alpha-\left\langle\boldsymbol{U}, \boldsymbol{e}_{l}^{*}(\boldsymbol{x})\right\rangle\right) \boldsymbol{e}_{l}^{*}(\boldsymbol{x})
$$

This means that according to Eq. (24), a sample $Z$ will fall in the failure region $F_{l}(\boldsymbol{x})$ and lead to ${ }^{253}$ $I_{F_{l}}(\boldsymbol{x}, \boldsymbol{Z})=1$. A schematic representation illustrating the capabilitiy of Eq. (24) for generating 254 failure samples is shown in Fig. $1 . \quad 255$

In time-dependent problems, there are several limit state functions associated with a certain 256 realization $\boldsymbol{x}$ at different time instants, i.e. $g_{Z}\left(\boldsymbol{x}, \boldsymbol{Z}, t_{l}\right), l=0, \ldots, m$ (see Eq. (12)). Taking ${ }^{257}$ this fact into account, the proposed importance sampling density function $H(\boldsymbol{Z} \mid \boldsymbol{x})$ is constructed 258 


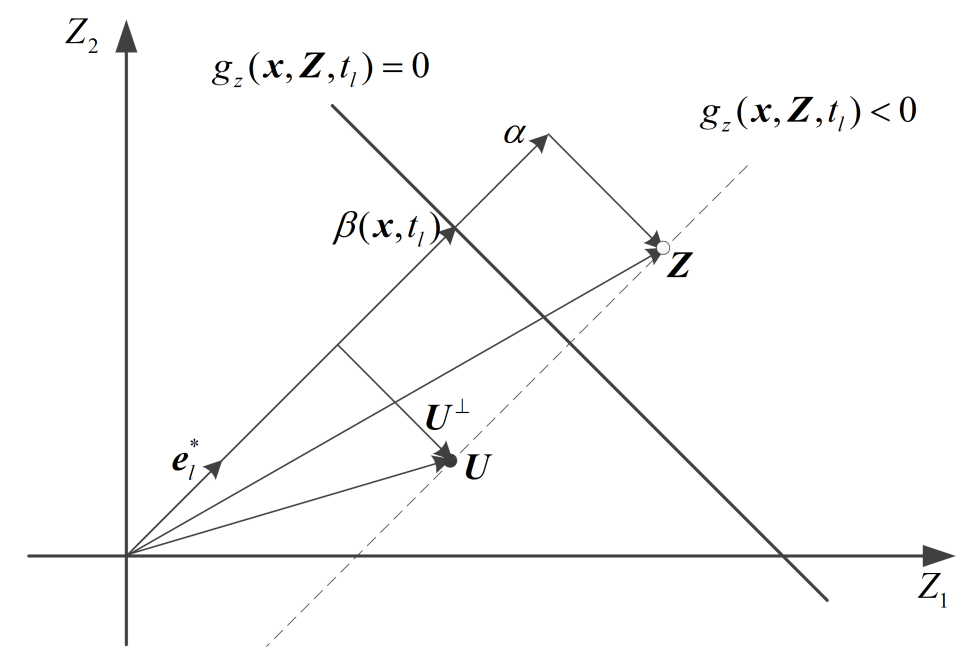

Figure 1: Schematic diagram of sampling of conditional $Z$.

based on a combination of individual optimal sampling functions $f\left(\boldsymbol{Z} \mid F_{l}(\boldsymbol{x})\right)$ which is given by 259 $[27]:$

$$
H(\boldsymbol{Z} \mid \boldsymbol{x})=\sum_{l=0}^{m} w_{l}(\boldsymbol{x}) f\left(\boldsymbol{Z} \mid F_{l}(\boldsymbol{x})\right)=\sum_{l=0}^{m} w_{l}(\boldsymbol{x}) \frac{\phi(\boldsymbol{Z}) I_{F_{l}}(\boldsymbol{x}, \boldsymbol{Z})}{\Phi\left[-\beta\left(\boldsymbol{x}, t_{l}\right)\right]}
$$

where $w_{l}(\boldsymbol{x}) \geq 0$ are the weights of individual sampling functions, which fulfill the conditions that ${ }^{261}$ $w_{l}(\boldsymbol{x}) \geq 0$ and $\sum_{l=1}^{m} w_{l}(\boldsymbol{x})=0$. In this contribution, the weights are chosen to be proportional to ${ }_{262}$ the probability content of $F_{l}(\boldsymbol{x})=\left\{g_{Z}\left(\boldsymbol{x}, \boldsymbol{Z}, t_{l}\right) \leq 0\right\}[39]$ :

$$
w_{l}(\boldsymbol{x})=\frac{P\left(F_{l}(\boldsymbol{x})\right)}{\sum_{s=0}^{m} P\left(F_{s}(\boldsymbol{x})\right)}=\frac{\Phi\left[-\beta\left(\boldsymbol{x}, t_{l}\right)\right]}{\sum_{s=0}^{m} \Phi\left[-\beta\left(\boldsymbol{x}, t_{s}\right)\right]}
$$

Substituting Eq. (26) into (25), the proposed importance sampling density function $H(\boldsymbol{Z} \mid \boldsymbol{x})$ is ${ }_{264}$ finally expressed by:

$$
H(\boldsymbol{Z} \mid \boldsymbol{x})=\phi(\boldsymbol{Z}) \frac{\sum_{l=0}^{m} I_{F_{l}}(\boldsymbol{x}, \boldsymbol{Z})}{\sum_{l=0}^{m} \Phi\left[-\beta\left(\boldsymbol{x}, t_{l}\right)\right]}
$$

\subsubsection{Estimation of the failure probability}

Using the newly introduced ISD $H(\boldsymbol{Z} \mid \boldsymbol{x})$ in Eq. (27) as the importance sampling density, the time-dependent failure probability in Eq. (13) is expressed as:

$$
\text { tine-dependent failure probability in Eq. (13) is expressed as. }
$$

$$
\begin{aligned}
P_{f}(0, T) & =\iint I_{F_{m}^{U}}\left[\boldsymbol{x}, t_{0}, \cdots, t_{m}, \boldsymbol{Z}\right] \frac{\phi(\boldsymbol{Z})}{H(\boldsymbol{Z} \mid \boldsymbol{x})} H(\boldsymbol{Z} \mid \boldsymbol{x}) f(\boldsymbol{x}) \mathrm{d} \boldsymbol{x} \mathrm{d} \boldsymbol{Z} \\
& =E\left[I_{F_{m}^{U}}\left(\boldsymbol{x}, t_{0}, \cdots, t_{m}, \boldsymbol{Z}\right) \frac{\phi(\boldsymbol{Z})}{H(\boldsymbol{Z} \mid \boldsymbol{x})}\right] \\
& =E\left[\frac{\sum_{l=0}^{m} \Phi\left[-\beta\left(\boldsymbol{x}, t_{l}\right)\right]}{\sum_{l=0}^{m} I_{F_{l}}(\boldsymbol{x}, \boldsymbol{Z})}\right]
\end{aligned}
$$


Note that $I_{F_{m}^{U}}\left[\boldsymbol{x}, t_{0}, \cdots, t_{m}, \boldsymbol{Z}\right]=1$ has been considered within Eq. (28). The reason is that 269 every sample $\boldsymbol{Z}$ generated from $H(\boldsymbol{Z} \mid \boldsymbol{x})$ is - by construction - located in at least one of the 270 failure domains $F_{l}(\boldsymbol{x})$ (see Eq. 24), and as $F_{l}(\boldsymbol{x}) \subset F_{m}^{U}$, thus $\boldsymbol{Z} \in F_{m}^{U}$ holds, leading to ${ }^{271}$ $I_{F_{m}^{U}}\left[\boldsymbol{x}, t_{0}, \cdots, t_{m}, \boldsymbol{Z}\right]=1$.

The expression for the failure probability as cast in Eq. (28) can be evaluated by means of 273 simulation. For that purpose, samples of the static random variables $\boldsymbol{x}^{(j)}: j=1, \ldots, N$ distributed ${ }^{274}$ according to $f(\boldsymbol{x})$ are generated in the first place. Then, samples of $\boldsymbol{Z}$ are generated such that 275 they follow $H(\boldsymbol{Z} \mid \boldsymbol{x})$, yielding a set of samples, $\left(\boldsymbol{x}^{(j)}, \boldsymbol{Z}^{(j)}\right), j=1, \ldots, N$ which is distributed as 276 $H(\boldsymbol{Z} \mid \boldsymbol{x}) f(\boldsymbol{x})$. Thus, $P_{f}(0, T)$ is estimated as:

$$
\hat{P}_{f}(0, T)=\frac{1}{N} \sum_{j=1}^{N} \frac{\sum_{l=0}^{m} \Phi\left[-\beta\left(\boldsymbol{x}^{(j)}, t_{l}\right)\right]}{\sum_{l=0}^{m} I_{F_{l}}\left(\boldsymbol{x}^{(j)}, \boldsymbol{Z}^{(j)}\right)}
$$

Obviously, the estimate $\hat{P}_{f}(0, T)$ is unbiased. The variance as well as the coefficient of variation ${ }^{278}$ (c.o.v., denoted as $\delta$ ) of the estimate $\hat{P}_{f}(0, T)$ can be obtained straightforwardly:

$$
\begin{gathered}
\operatorname{Var}\left[\hat{P}_{f}(0, T)\right] \approx \frac{1}{N-1}\left\{\frac{1}{N} \sum_{j=1}^{N}\left[\frac{\sum_{l=0}^{m} \Phi\left[-\beta\left(\boldsymbol{x}^{(j)}, t_{l}\right)\right]}{\sum_{l=0}^{m} I_{F_{l}}\left(\boldsymbol{x}^{(j)}, \boldsymbol{Z}^{(j)}\right)}\right]^{2}-\hat{P}_{f}^{2}(0, T)\right\} \\
\delta\left[\hat{P}_{f}(0, T)\right] \approx \frac{\sqrt{\operatorname{Var}\left[\hat{P}_{f}(0, T)\right]}}{\hat{P}_{f}(0, T)}
\end{gathered}
$$

In conclusion, the proposed approach utilizes the information on the 'conditional linear' rela- ${ }^{281}$ tionship between the response and the applied load. Based on this fact, an efficient importance ${ }_{282}^{2}$ sampling density function is constructed based on analytical investigation of the failure regions. It 283 can be seen from Eq. (29) that as the probability estimator is simulation-based, there is no need 284 for fitting a distribution, or conducting approximate analytical calculations. Hence, the accuracy 285 can be guaranteed as the simulation proceeds, i.e. as the number of samples increases. In the ${ }^{286}$ following numerical applications, it is shown that the proposed approach exhibits excellent effi- ${ }^{287}$ ciency, and that the proposed two-step designed importance sampling density function is highly ${ }_{288}$ rewarding.

\subsection{Summary of the proposed approach}

The approach for estimating the failure probability of a time-variant structure subject to time- ${ }^{291}$ variant load proposed in this section is summarized as follows. 
1. Stochastic processes are represented by spectral decomposition as in Eq. (8), and the equiv- ${ }^{293}$ alent composite limit functions are obtained according to Eq. (12). 294

2. Generate samples $\boldsymbol{x}^{(j)}, j=1, \ldots, N$ following $f(\boldsymbol{x}) . \quad 295$

3. Draw a set of samples $\boldsymbol{Z}^{(j)}, j=1, \ldots, N$ according to the proposed ISD given by Eq. (27). ${ }^{296}$ Specifically: 297 (a) Draw an index $l$ within the discrete set $\{0, \ldots, m\}$ with probability proportional to 298 $w_{l}\left(\boldsymbol{x}^{(j)}\right)$.

(b) Simulate $\boldsymbol{U}$ as a $n$-dimensional standard Gaussian vector with independent components, 300 and $u$ as uniform variable on $[0,1]$. Compute 301

$$
\alpha=\Phi^{-1}\left[u+(1-u) \Phi\left(\beta\left(\boldsymbol{x}^{(j)}, t_{l}\right)\right)\right]
$$

or

$$
\alpha=-\Phi^{-1}\left[(1-u) \Phi\left(-\beta\left(\boldsymbol{x}^{(j)}, t_{l}\right)\right)\right]
$$

and set

4. Based on the generated samples set $\left(\boldsymbol{x}^{(j)}, \boldsymbol{Z}^{(j)}\right), j=1, \ldots, N$, compute the failure proba- 304 bility estimator according to Eq. (29) as well as the coefficient of variation of the estimator 305 according to Eq. (31). 306

\section{Examples}

In this section, examples are given to illustrate the performance of the proposed method in 308 terms of accuracy and efficiency. Direct Monte Carlo simulation (MCS) and Subset simulation 309 (SS) are used for comparison. Note that the unit coefficient of variation (c.o.v.) $\Delta$ is calculated 310 in all examples considered. Since any simulation algorithm for estimating failure probabilities has 311 a c.o.v. of the form $\delta=\Delta / \sqrt{N}$ [27], the 'unit c.o.v.' $\Delta$ is adopted as a measure of efficiency 312 which is inherent to the algorithm. That is, it is in theory invariant to the accuracy achieved and 313 the computational effort spent, where smaller values of $\Delta$ correspond to a higher computational 314 efficiency. In addition, the computational cost is measured in terms of the number of samples $N \quad 315$ considered for the evaluation of the failure probability. 316

For Examples 1 to 3, a time period of $[0,10]$ years is considered, and a constant time interval 317 $\Delta t=0.1$ year is adopted to discretize the time interval in the calculation. As such, a number 318 
of $m=101$ discrete time instants are obtained, i.e., $t_{l}=l \Delta t, l=0, \ldots, m$. Also, the number of 319 identified dominating eigenfunctions in $\mathrm{K}-\mathrm{L}$ expansion is fixed at $k=30$ which has been found 320 to be reasonable by carrying out some numerical validations. For Example 4, the time interval 321 and the number of expansion terms for the stochastic process are considered as $\Delta t=0.25$ year 322 and $k=30$, respectively, as it involves finite element analysis. Such setting is used in order to 323 alleviate the computation burden associated with MCS.

\subsection{Example 1: A two-bar frame}

A two-bar frame shown in Fig. 2 is considered for the first example. It has been investigated 326 in [40] and [41] (among others) and is modified to suit the purposes of this work. This frame is 327 subjected to a dynamic force $F(t)$ which is described by a stochastic process. The yield strength of 328 the bar A degrades with time, i.e. $S(\mathrm{t})=S_{0} e^{(-0.05 t)}$, in which $S_{0}$ is the initial yield strength of the ${ }_{329}$ bar. Structural failure is defined as the maximum stress of the bar exceeding the corresponding 330 yield strength. Thus, the limit state function of this two-bar frame is expressed as follows. 331

$$
g(\boldsymbol{x}, t, \boldsymbol{Y}(t))=\pi l_{2} D^{2} S(t)-4 F(t) \sqrt{l_{1}^{2}+l_{2}^{2}}
$$

where $\boldsymbol{x}=\left[D, l_{1}, l_{2}, S_{0}\right]$ is the vector of structural random variables; $\boldsymbol{Y}(t)=F(t)$ is the vector ${ }_{332}$ of stochastic load; $t \in[0,10]$ year. Distribution information of the input parameters is listed in 333 Table 1. As can be noted from this table, the load is described by a Gaussian stochastic process 334 with mean $\mu=2.2 \times 10^{6}(\mathrm{~N})$ and standard deviation $\sigma=2.2 \times 10^{5}(\mathrm{~N})$. The autocorrelation of 335 the process is given by a squared exponential correlation function with correlation length equal to 336 1 year.

Table 1: Information of variables and parameters for two-bar frame (Example 1)

\begin{tabular}{ccccc}
\hline Parameter & Distribution & Mean & Standard deviation & Auto-correlation function \\
\hline$D(\mathrm{~m})$ & Normal & 0.2 & $2 \times 10^{-3}$ & - \\
$l_{1}(\mathrm{~m})$ & Normal & 0.4 & $4 \times 10^{-3}$ & - \\
$l_{2}(\mathrm{~m})$ & Normal & 0.3 & $3 \times 10^{-3}$ & - \\
$S_{0}(\mathrm{~Pa})$ & Lognormal & $2.5 \times 10^{8}$ & $2.5 \times 10^{6}$ & - \\
$F(t)(\mathrm{N})$ & Gaussian process & $2.2 \times 10^{6}$ & $2.2 \times 10^{5}$ & $e^{-\tau^{2}}$ \\
\hline
\end{tabular}

The proposed approach is applied to solve this problem. In addition, Subset simulation and 338 Direct Monte Carlo simulation are also applied for comparison. The details of the results obtained 339 


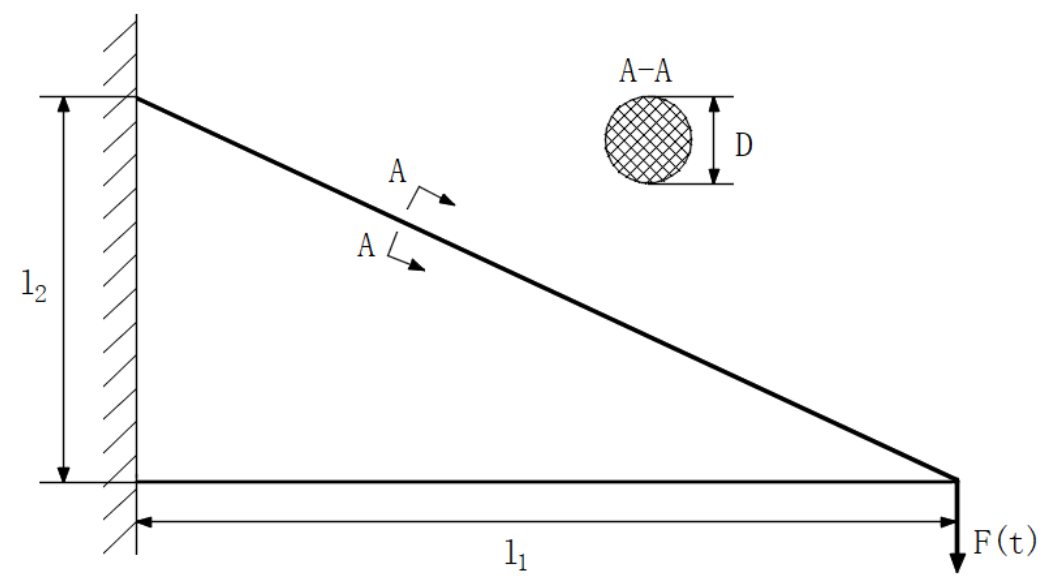

Figure 2: A two-bar frame

Table 2: Results by different methods for two-bar frame beam (Example 1)

\begin{tabular}{ccccc}
\hline Methods & $P_{f}(0, T)$ & c.o.v. & $N$ & Unit c.o.v. \\
\hline Proposed approach & $3.01 \times 10^{-3}$ & 0.12 & 100 & 1.2 \\
SS & $3.79 \times 10^{-3}$ & 0.20 & 3000 & 10.9 \\
MCS & $3.05 \times 10^{-3}$ & 0.02 & $10^{6}$ & 18.1 \\
\hline
\end{tabular}
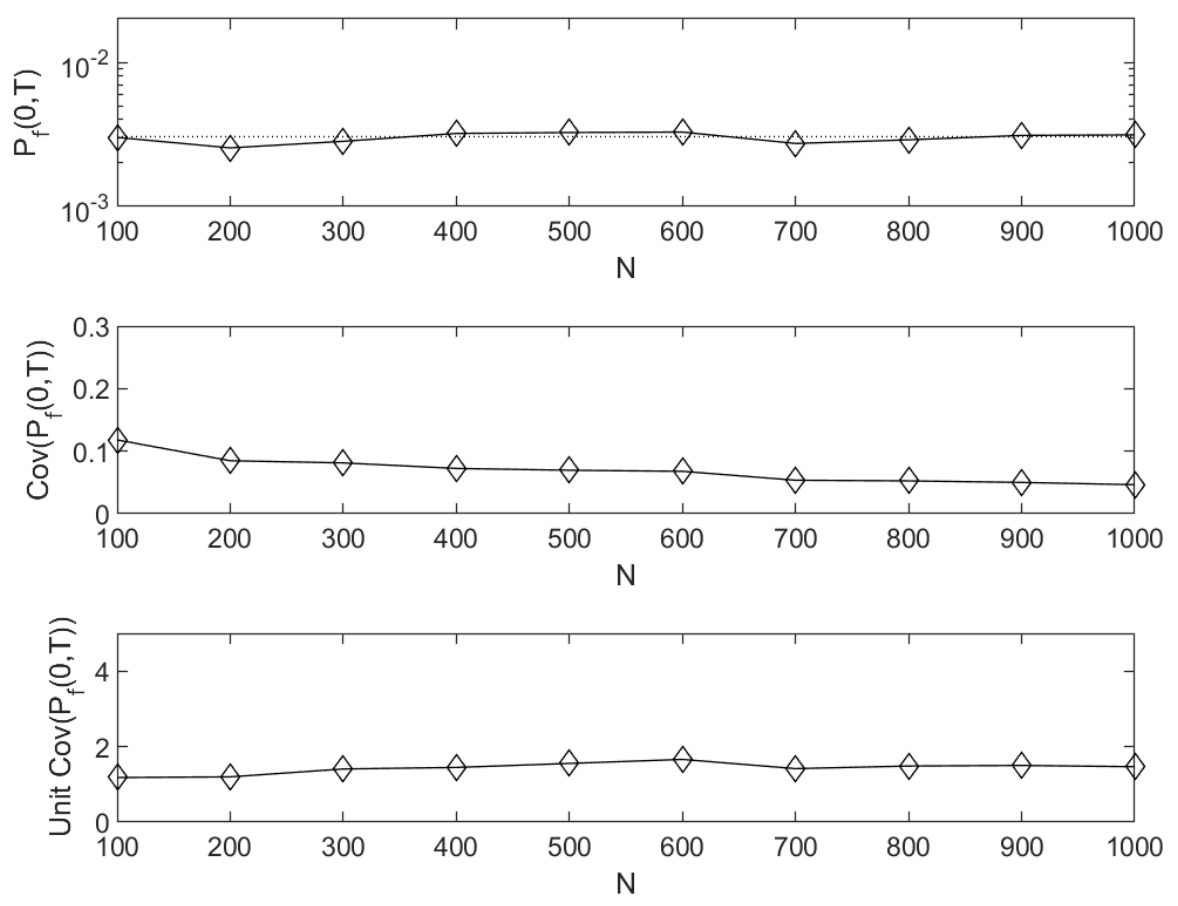

Figure 3: Evolution of different estimators with respect to the number of samples employing the proposed approach (Example 1). 

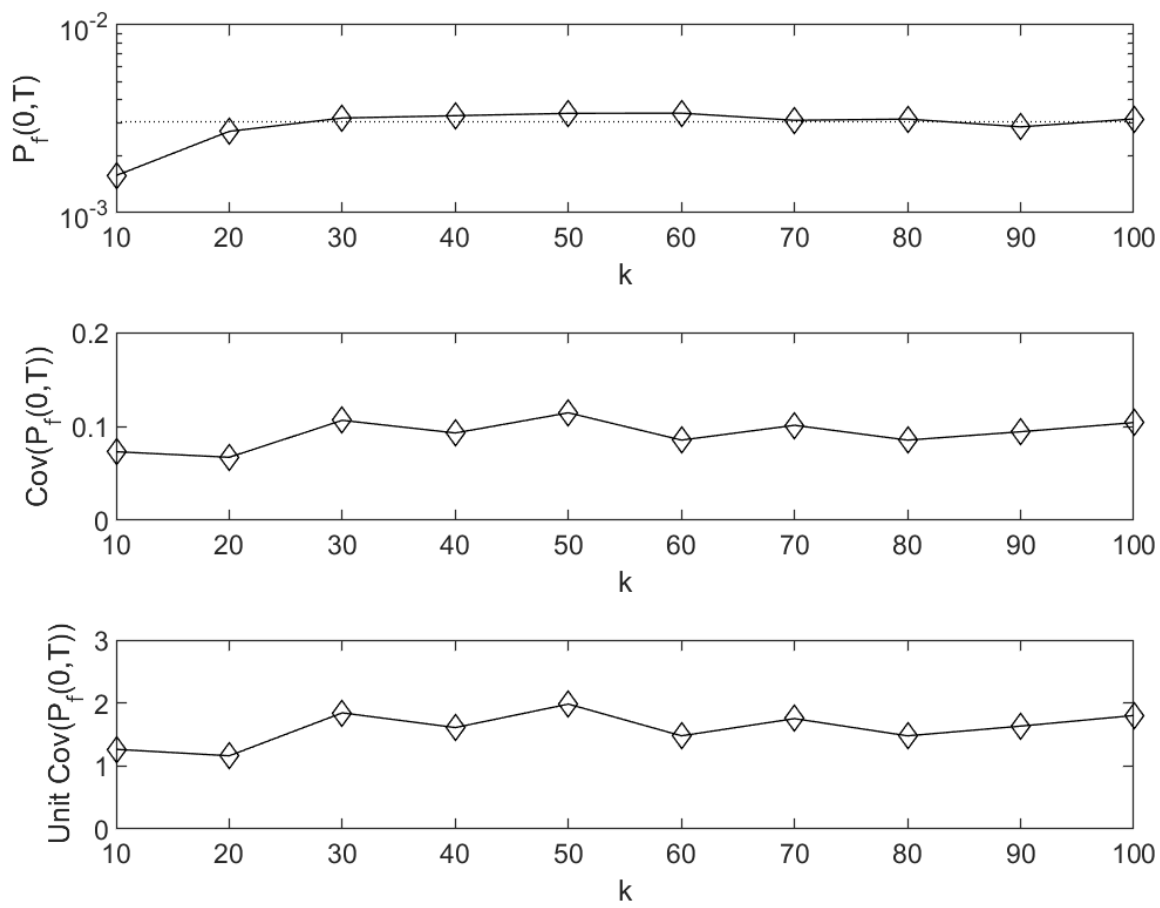

Figure 4: Evolution of different estimators with respect to the number of K-L expansion terms employing the proposed approach (Example 1).

with each approach are listed in Table 2, which includes the estimate of failure probability, the 340 c.o.v. of estimate, the number of samples used $N$, and the unit c.o.v. of the method. It can be ${ }^{341}$ seen from the table that the results that are obtained by these methods agree very well with each ${ }_{342}$ other. Note that the proposed approach has been applied with only 100 samples, leading to a ${ }^{343}$ c.o.v. of around 0.10. On the other hand, Subset simulation demanded 3000 samples, whereas the 344 c.o.v. is 0.20 . As such, it is clear that the proposed approach is highly efficient. Such conclusion 345 is reinforced when examining the value of the unit c.o.v., which is an index of efficiency for a 346 simulation method. It can be seen that the proposed method owns the smallest unit c.o.v., which 347 is nearly $1 / 20$ of that of MCS and 1/10 of that of Subset simulation for this example. Due to the ${ }^{348}$ small unit c.o.v., the proposed approach can significantly reduce the computation cost. 349

In order to investigate the performance of the proposed method more clearly, several runs with 350 different number of samples are carried out. Fig. 3 shows the corresponding results obtained by 351 the proposed method as a function of the number of samples used. It can be seen from the figure ${ }_{352}$ that, even when a small number of samples (100) is used, the proposed approach can produce ${ }_{353}$ quite accurate estimates. Furthermore, when the number of samples increases from 100 to 1000, 354 
the c.o.v. of the failure probability estimate gradually decreases from about 0.1 to about 0.04 . At 355 the same time, the unit c.o.v. fluctuates between approximate 1 and 2 . 356

Also, the sensitivity of the probability with respect to the number of terms $(k)$ in the K-L 357 expansion is shown in Fig. (4). It can be seen from this figure that 1) the failure probability is 358 first underestimated and becomes stable when $k$ is larger than 30; 2) the c.o.v. and unit c.o.v. 359 of the estimate fluctuate when $k>30$, implying that the efficiency of the proposed approach 360 seems insensitive to the number of terms $k$, under the condition that $k$ is large enough such that 361 the error is controlled appropriately. This shows that the method is insensitive to the number of 362 transformed variables in this example ( according to Eq. (12)). 363

In conclusion, from the figure and table, the obtained results show that, for this kind of 364 problem, the proposed method exhibits high efficiency when compared to other existing methods. 365 The reason for this behavior is that the proposed method exploits the linearity with respect to 366 the stochastic process.

\subsection{Example 2: Composite beam}

This second example involves the composite beam shown in Fig.5, which has been borrowed from [42] and [43] with appropriate modifications. The beam possesses a cross section of width $A(\mathrm{~mm})$ and height $B(\mathrm{~mm})$ while its total length is $L(\mathrm{~mm})$. Its Young's modulus is denoted as $E_{w}(t)(\mathrm{GPa})$. The beam has attached on its bottom face an aluminum plate with Young's 372 modulus $E_{a}(t)(\mathrm{GPa})$, whose cross section is $C(\mathrm{~mm})$ wide by $D(\mathrm{~mm})$ high. Along the beam, six 373 time-dependent stochastic loads, $P_{1}(t), P_{2}(t), P_{3}(t), P_{4}(t), P_{5}(t)$ and $P_{6}(t)(\mathrm{kN})$ are applied at six $\quad 374$ different locations, $L_{1}, L_{2}, L_{3}, L_{4}, L_{5}$ and $L_{6}(\mathrm{~mm})$. Failure is defined whenever the maximum 375 bending normal stress of the beam exceeds the allowable tensile stress (yield strength) $S(t)$. The 376 limit state function is then given by

$$
g(\boldsymbol{x}, t, \boldsymbol{Y}(t))=S(t)-\frac{\left[\frac{L_{3}}{L} \sum_{i=1}^{6} P_{i}(t)\left(L-L_{i}\right)-\sum_{i=1}^{2} P_{i}(t)\left(L_{k}-L_{i}\right)\right] d(\boldsymbol{x})}{W(\boldsymbol{x})}
$$

where $\boldsymbol{Y}(t)=\left[P_{1}(t), P_{2}(t), P_{3}(t), P_{4}(t), P_{5}(t), P_{6}(t)\right]$ is the vector of stochastic processes;

$$
\begin{gathered}
d(\boldsymbol{x})=\frac{0.5 A B^{2}+D C(B+D) E_{a}(t) / E_{w}(t)}{A B+D C E_{a} / E_{w}} \\
W(\boldsymbol{x})=\frac{A B^{3}}{12}+A B\left[d(\boldsymbol{x})-\frac{B}{2}\right]^{2}+\frac{C D^{3} E_{a}(t)}{12 E_{w}(t)}+\frac{C D E_{a}(t)}{E_{w}(t)}\left[\frac{D}{2}+B-d(\boldsymbol{x})\right]^{2}
\end{gathered}
$$

and

$$
S(t)=S_{0} e^{-0.01 t}
$$




$$
\begin{gathered}
E_{a}(t)=E_{a 0}[1-0.1 \log (t / 2+1)] \\
E_{w}(t)=E_{w 0}[1-0.3 \log (t+1)]
\end{gathered}
$$

are three random processes related with structural parameters' deterioration. In this context, 383 $S_{0}$ is the initial yield strength, and $E_{a 0}, E_{w 0}$ are the initial Young's moduli of the beam and 384 the aluminum plate, respectively. There are 14 random variables and 6 stochastic load process 385 considered in this example. The distribution information of these parameters is given in Table $3 . \quad 386$
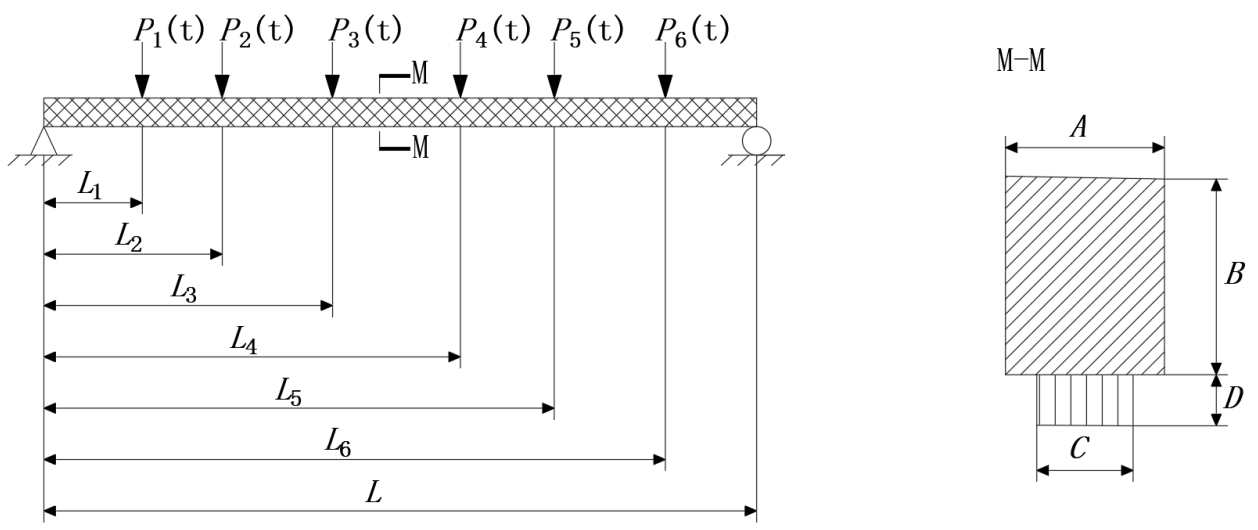

Figure 5: A composite beam.

In this example, six stochastic processes are considered simultaneously. The proposed ap- 387 proach, as well as Subset simulation and Direct Monte Carlo simulation are applied to solve this 388 problem. Table 4 exhibits the obtained results by these methods, showing consistency between 389 methods with respect to the failure probability estimates. In this case, MCS shows a relatively 390 low efficiency, where $10^{6}$ samples are used and the unit c.o.v is 103.7. Subset simulation produces 391 a probability estimate with a relatively large c.o.v. (0.29). The proposed approach has been 392 carried out with 500 samples and produces an estimate with c.o.v. around 0.10. In addition, the 393 proposed method owns the smallest unit c.o.v. which is nearly $1 / 8$ of that of MCS and 1/40 of 394 that of Subset simulation in this example. The advantage on efficiency of the proposed approach 395 is obvious.

\subsection{Example 3: Ten-bar truss structure}

This example involves a ten-bar aluminum truss, which corresponds to a modified version of 398 the problem considered in [44] and [45] in order to suit the purposes of this contribution. The truss 399 
Table 3: Information parameters for composite beam (Example 2)

\begin{tabular}{|c|c|c|c|c|}
\hline Parameter & Distribution & Mean & Standard deviation & Autocorrelation function \\
\hline$A(\mathrm{~mm})$ & Normal & 100 & 1 & - \\
\hline$B(\mathrm{~mm})$ & Normal & 200 & 2 & - \\
\hline$C(\mathrm{~mm})$ & Normal & 80 & 0.8 & - \\
\hline$D(\mathrm{~mm})$ & Normal & 20 & 0.2 & - \\
\hline$L_{1}(\mathrm{~mm})$ & Normal & 200 & 2 & - \\
\hline$L_{2}(\mathrm{~mm})$ & Normal & 400 & 4 & - \\
\hline$L_{3}(\mathrm{~mm})$ & Normal & 600 & 6 & - \\
\hline$L_{4}(\mathrm{~mm})$ & Normal & 800 & 8 & - \\
\hline$L_{5}(\mathrm{~mm})$ & Normal & 1000 & 10 & - \\
\hline$L_{6}(\mathrm{~mm})$ & Normal & 1200 & 12 & - \\
\hline$L(\mathrm{~mm})$ & Normal & 1400 & 14 & - \\
\hline$E_{a 0}(\mathrm{GPa})$ & Extreme value & 70 & 0.7 & - \\
\hline$E_{w 0}(\mathrm{GPa})$ & Extreme value & 8.75 & 0.0875 & - \\
\hline$S_{0}(\mathrm{GPa})$ & Lognormal & $2.7 \times 10^{-2}$ & $2.7 \times 10^{-4}$ & - \\
\hline$P_{1}(t)(\mathrm{kN})$ & Gaussian process & 15 & 1.5 & $\cos (\pi \tau)$ \\
\hline$P_{2}(t)(\mathrm{kN})$ & Gaussian process & 15 & 1.5 & $\cos (\pi \tau)$ \\
\hline$P_{3}(t)(\mathrm{kN})$ & Gaussian process & 15 & 1.5 & $\cos (\pi \tau)$ \\
\hline$P_{4}(t)(\mathrm{kN})$ & Gaussian process & 15 & 1.5 & $\cos (\pi \tau)$ \\
\hline$P_{5}(t)(\mathrm{kN})$ & Gaussian process & 15 & 1.5 & $\cos (\pi \tau)$ \\
\hline$P_{6}(t)(\mathrm{kN})$ & Gaussian process & 15 & 1.5 & $\cos (\pi \tau)$ \\
\hline
\end{tabular}

Table 4: Results by different methods for composite beam (Example 2)

\begin{tabular}{ccccc}
\hline Methods & $P_{f}(0, T)$ & c.o.v. & $N$ & Unit c.o.v. \\
\hline Proposed approach & $9.1 \times 10^{-5}$ & 0.11 & 500 & 2.59 \\
SS & $8.6 \times 10^{-5}$ & 0.29 & 5000 & 20.6 \\
MCS & $9.3 \times 10^{-5}$ & 0.10 & $10^{6}$ & 103.7 \\
\hline
\end{tabular}


is shown in Fig. 6. In this example, the length of the vertical and horizontal bars is $L$, the modulus 400 of elasticity is $E(t)$, and the cross-sectional area of its members is denoted as $A_{j} j=1,2, \ldots, 10$. 401 These quantities are all modelled as basic random variables. This truss is subjected to two vertical 402 stochastic process loads $F_{1}(t)$ and $F_{2}(t)$, and a horizontal stochastic process load $F_{3}(t)$. The limit ${ }_{403}$ state function is cast as the difference between the allowable displacement $d(t)$ and the vertical 404 displacement $\delta_{2}$ of joint 2 , that is:

$$
g(\boldsymbol{x}, t, \boldsymbol{Y}(t))=d(t)-\left(\sum_{i=1}^{6} \frac{N_{i} N_{i}^{0}}{A_{i}}+\sqrt{2} \sum_{i=7}^{10} \frac{N_{i} N_{i}^{0}}{A_{i}}\right) \frac{L}{E(t)}
$$

where

$$
\begin{gathered}
d(t)=d_{0} e^{(-0.05 t)} \\
E(t)=E_{0}[1-0.1 \log (t+1)]
\end{gathered}
$$

are two stochastic processes related with structural deterioration, respectively; $\boldsymbol{x}=\left[A_{1}, \cdots, A_{10}, \quad 408\right.$ $\left.L, E_{0}, d_{0}\right]$ is the vector of basic random variables; $N_{j}(j=1,2, \ldots, 10)$ are the axial forces which 409 can be obtained from the equilibrium and compatibility equations:

where

$$
\left\{\begin{array}{l}
N_{1}=F_{2}-\sqrt{2} N_{8} / 2 \\
N_{2}=-\sqrt{2} N_{10} / 2 \\
N_{3}=-F_{1}(t)-2 F_{2}(t)+F_{3}(t)-\sqrt{2} N_{8} / 2 \\
N_{4}=-F_{2}(t)+F_{3}(t)-\sqrt{2} N_{10} / 2 \\
N_{5}=-F_{2}(t)-\sqrt{2} N_{8} / 2-\sqrt{2} N_{10} / 2 \\
N_{6}=-\sqrt{2} N_{10} / 2 \\
N_{7}=\sqrt{2}\left(F_{1}(t)+F_{2}(t)\right)+N_{8} \\
N_{8}=\left(a_{22} b_{1}-a_{12} b_{2}\right) /\left(a_{11} a_{22}-a_{12} a_{21}\right) \\
N_{9}=\sqrt{2} F_{2}(t)+N_{10} \\
N_{10}=\left(a_{11} b_{2}-a_{21} b_{1}\right) /\left(a_{11} a_{22}-a_{12} a_{21}\right)
\end{array}\right.
$$

$\left\{\begin{array}{l}a_{11}=\left(1 / A_{1}+1 / A_{3}+1 / A_{5}+2 \sqrt{2} / A_{7}+2 \sqrt{2} / A_{8}\right) L /(2 E) \\ a_{12}=a_{21}=L /\left(2 A_{5} E\right) \\ a_{22}=\left(1 / A_{2}+1 / A_{4}+1 / A_{6}+2 \sqrt{2} / A_{9}+2 \sqrt{2} / A_{10}\right) L /(2 E) \\ b_{1}=\left(F_{2}(t) / A_{1}-\left(F_{1}(t)+2 F_{2}(t)-F_{3}(t)\right) / A_{3}-F_{2}(t) / A_{5}-2 \sqrt{2}\left(F_{1}(t)+F_{2}(t)\right) / A_{7}\right) \sqrt{2} L /(2 E) \\ b_{2}=\left(\sqrt{2}\left(F_{3}(t)-F_{2}(t)\right) / A_{4}-\sqrt{2} F_{2}(t) / A_{5}-4 F_{2}(t) / A_{9}\right) L /(2 E)\end{array}\right.$ 
where $\boldsymbol{Y}(t)=\left[F_{1}(t), F_{2}(t), F_{3}(t)\right]$ is the vectors of load stochastic processes; $N_{i}^{0}$ is obtained by ${ }^{412}$ assuming $F_{1}=F_{3}=0$ and $F_{2}=1$ in Eq. 45 .

The information of basic random variables and the stochastic processes is given in Table $5 . \quad{ }_{414}^{41}$

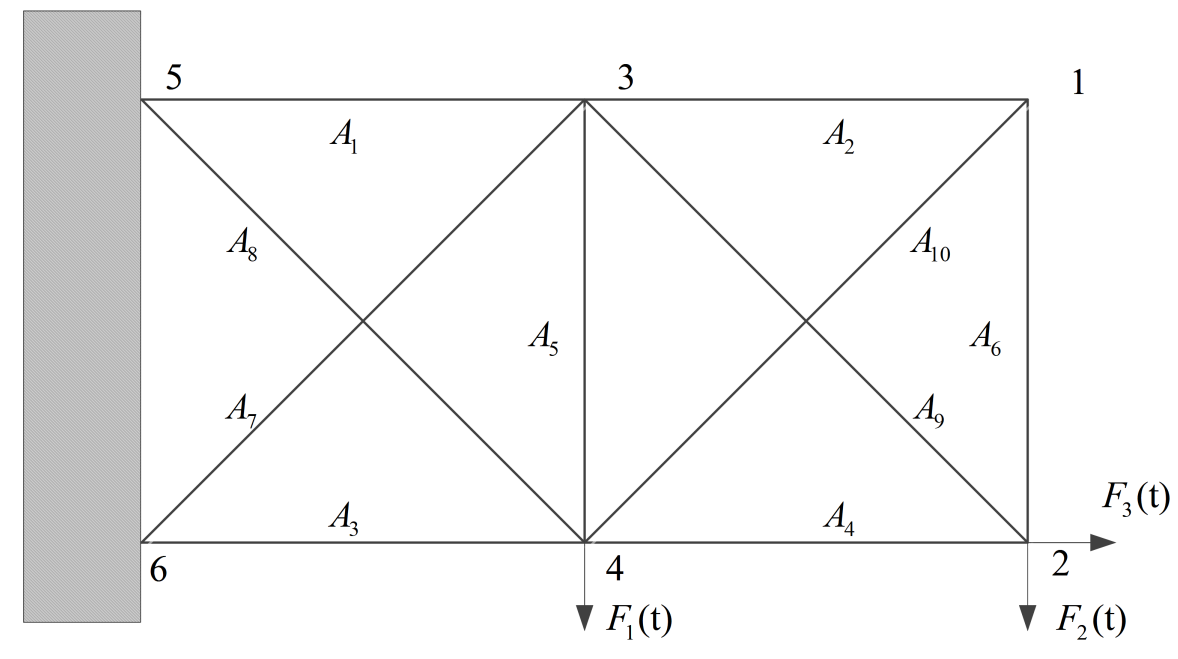

Figure 6: Ten-bar truss structure.

Table 5: Information of variables and parameters for ten-bar truss (Example 3)

\begin{tabular}{lllll}
\hline Parameter & Distribution & Mean & Standard deviation & Autocorrelation function \\
\hline$A_{1}, \cdots, A_{10}($ in $)$ & Normal & 10 & 0.1 & - \\
$L(\mathrm{in})$ & Normal & 360 & 3 & - \\
$E_{0}(\mathrm{ksi})$ & Normal & $1.5 \times 10^{4}$ & $1.5 \times 10^{2}$ & - \\
$d_{0}(\mathrm{in})$ & Lognormal & 5 & 0.05 & - \\
$F_{1}(t)(\mathrm{kip})$ & Gaussian process & 100 & 10 & $e^{-\tau^{2}}$ \\
$F_{2}(t)(\mathrm{kip})$ & Gaussian process & 120 & 12 & $e^{-\tau^{2}}$ \\
$F_{3}(t)(\mathrm{kip})$ & Gaussian process & 400 & 40 & $e^{-\tau^{2}}$ \\
\hline
\end{tabular}

In this example, three stochastic processes are considered. The proposed approach, Subset ${ }_{415}$ simulation and Monte Carlo simulation are applied to solve this problem. Table 6 shows the ${ }_{416}$ obtained results by these methods. It can be seen from the table that the results by these methods ${ }_{417}$ are consistent with each other. Note that the failure probability is quite small $\left(\approx 10^{-4}\right)$. MCS 418 shows a low efficiency with unit c.o.v. bigger than 100; SS also obtained an estimate with relatively ${ }_{419}$ large c.o.v. of 0.25 (1000 samples were considered for each level). The proposed approach has 420 been carried out with only 100 samples and obtains a estimate with c.o.v. below 0.10. Clearly, 421 
Table 6: Results by different methods for ten-bar truss (Example 3)

\begin{tabular}{ccccc}
\hline Methods & $P_{f}(0, T)$ & c.o.v. & Total number of samples & Unit c.o.v. \\
\hline Proposed approach & $8.2 \times 10^{-5}$ & 0.08 & 100 & 0.8 \\
SS & $6.5 \times 10^{-5}$ & 0.25 & 5000 & 22.5 \\
MCS & $8.9 \times 10^{-5}$ & 0.11 & $10^{6}$ & 105.9 \\
\hline
\end{tabular}

the proposed method owns the smallest unit c.o.v. which is nearly $1 / 100$ of that of MCS and $1 / 25 \quad 422$ of that of Subset simulation in this example. $\quad 423$

\subsection{Example 4: Bracket structure $\quad 424$}

This example involves a bracket structure. Its 3-D finite element model is shown schematically $\quad 425$ in Fig. 7. The rear face of the bracket is fixed, and a distributed stochastic process load $F(t) \quad{ }_{426}$ is applied in the negative vertical direction over the front face. In this example, the maximum ${ }_{427}$ allowable deflection of the tip of the bracket in the vertical direction is $d_{0}$, which is characterized ${ }_{428}$ as a random variable. The limit state function is defined as:

$$
g(\boldsymbol{x}, t, \boldsymbol{Y}(t))=d_{0}-\delta_{\max }(E(t), \gamma, P(t))
$$

where $\gamma$ is Poisson's ratio; $\delta_{\max }(\cdot)$ is the maximum displacement of the bracket, which is determined 430 through a finite element analysis in Matlab utilizing the Partial Differential Equation (PDE) ${ }_{431}$ toolbox; $E(t)$ is the Young's modulus, which is a random time-variant process given by 432

$$
E(t)=E_{0}(1-0.2 \log (t+1))
$$

The distribution information of the parameters is given in Table 7.

In this linear elastic problem, the response (maximum vertical displacement) has a linear ${ }_{434}$ relationship with load (provided that strains and stresses are below the elastic limit). Therefore, 435 the proposed approach can be applied, even though it actually involves an implicit limit state ${ }_{436}$ function.

Table 8 shows the results obtained by different reliability methods. It can be seen from the ${ }_{438}$ table that the results by these methods are consistent with each other. The proposed approach 439 has been carried out with only 30 samples and obtains an estimate of the failure probability 440 with a c.o.v. around 0.10. Figure 8 illustrates the evolution of the estimates generated with the ${ }_{441}$ proposed approach, confirming that 30 samples suffice for an accurate analysis. As in the previous ${ }_{442}$ 
examples, the proposed method owns the smallest unit c.o.v. which is nearly $1 / 24$ and $1 / 15$ of 443 those associated with MCS and Subset simulation, respectively. The advantage on efficiency of 444 the proposed approach is remarkable, as it comprises an implicit LSF whose evaluation is time- ${ }_{445}$ consuming because of the finite element model.
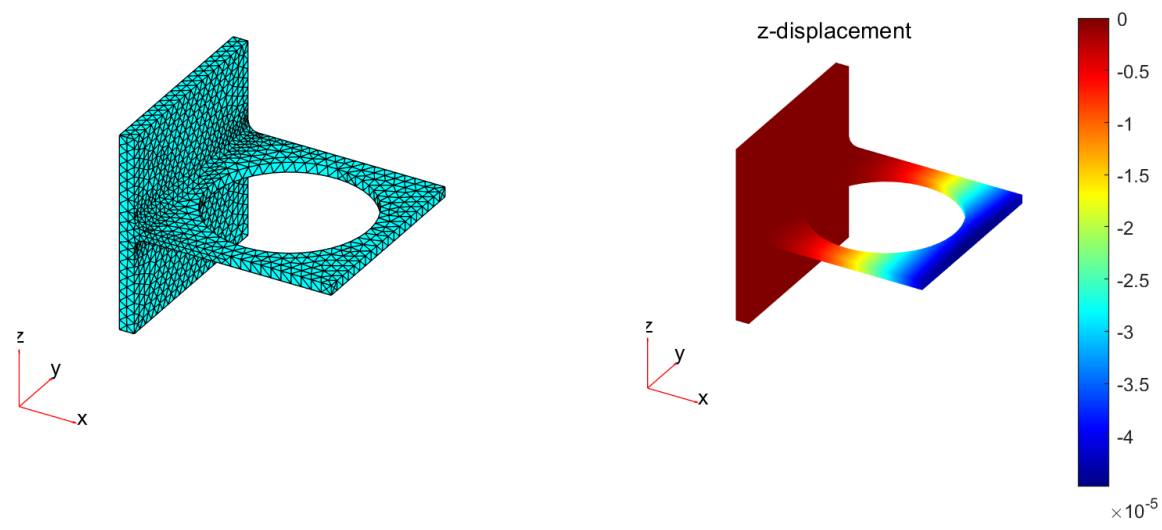

Figure 7: The finite model and response of bracket structure.

Table 7: Information of variables and parameters for bracket structure (Example 4)

\begin{tabular}{lllll}
\hline Parameter & Distribution & Mean & Standard deviation & Autocorrelation function \\
\hline$E_{0}(\mathrm{GPa})$ & Lognormal & 200 & 2 & - \\
$\gamma$ & Lognormal & 0.3 & 0.03 & - \\
$d_{0}(\mathrm{~m})$ & Extreme value & $2.0 \times 10^{-4}$ & $2.0 \times 10^{-6}$ & - \\
$P(t)\left(\mathrm{N} / \mathrm{m}^{2}\right)$ & Gaussian process & $1.8 \times 10^{4}$ & $1.8 \times 10^{3}$ & $e^{-\tau^{2}}$ \\
\hline
\end{tabular}

Table 8: Results by different methods for bracket example (Example 4)

\begin{tabular}{ccccc}
\hline Methods & $P_{f}(0, T)$ & c.o.v. & Total number of samples & Unit c.o.v. \\
\hline Proposed approach & $3.4 \times 10^{-3}$ & 0.12 & 30 & 0.68 \\
SS & $5.1 \times 10^{-3}$ & 0.19 & 3000 & 10.3 \\
MCS & $3.7 \times 10^{-3}$ & 0.12 & $2 \times 10^{4}$ & 16.4 \\
\hline
\end{tabular}

\section{Conclusions}

A new efficient importance sampling method has been proposed to estimate the reliability of ${ }_{448}$ a time-variant structure subject to time-variant load, where the limit state function includes ran- ${ }_{449}$ dom variables, structural degradation parameter processes and Gaussian stochastic load processes. $\quad 450$ 

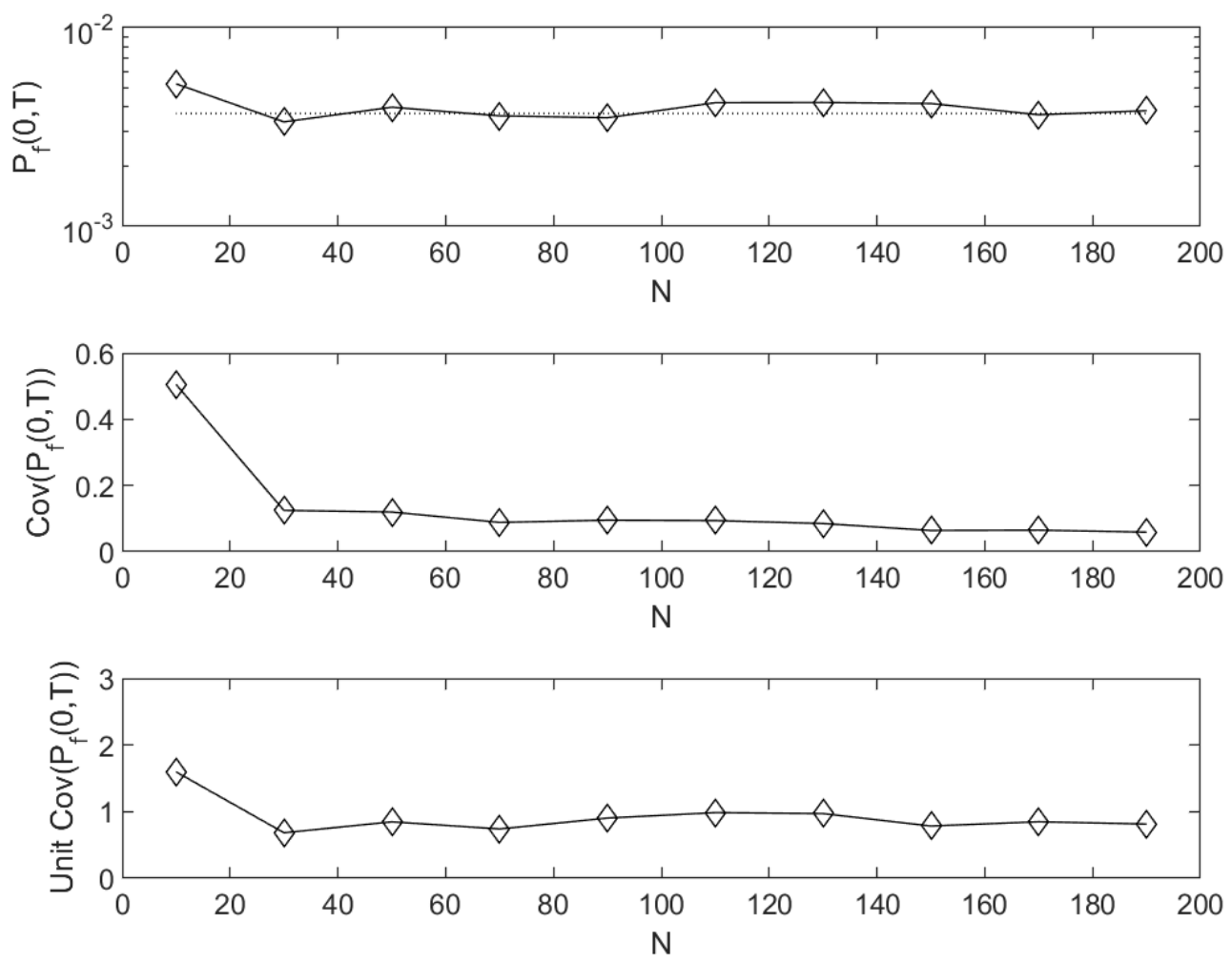

Figure 8: Evolution of estimators with respect to the number of samples by the proposed approach (Example 4). 
This approach first utilizes the series expansion methods to discretize the stochastic process and 451 transforms the time-variant problem into a series system. Then, a two-step importance sampling 452 density function is constructed to carry out the reliability analysis by means of simulation. Ex- ${ }_{453}$ amples have been presented to investigate the performance of the proposed approach. It is shown ${ }_{454}$ that the proposed approach has advantages in the following aspects: (1) It owns high efficiency, 455 as it can obtain a reliable estimate of the probability with only a hundred of samples in the given 456 examples. (2) The accuracy of the failure probability evaluated by proposed approach is relatively ${ }_{457}$ insensitive to the magnitude of the probability itself. (3) No approximate analytical calculation ${ }_{458}$ is involved.

Note that the application of the proposed approach is limited to problems involving first order $\quad{ }_{460}$ stochastic processes. That is, the performance of the structural system must exhibit a linear ${ }_{461}$ dependency with respect to the stochastic load for a fixed time. It is expected that it can also ${ }_{462}$ be applied to weakly non-linear stochastic processes. It should also be recalled that the proposed ${ }_{463}$ approach is applicable for those cases where the limit state function is linear with respect to the ${ }_{464}$ loading terms. Another aspect that should be also pointed out is that the proposed approach is 465 based on composite LSF idea which is sensitive to the time discretization. Then, a smaller interval ${ }_{466}$ will result in an increased number of composite LSFs, which involves bigger computational burden. $\quad{ }_{467}$ It is also found that the efficiency (indicated by the unit c.o.v.) may vary for different problems. ${ }^{468}$ Generally speaking, the proposed approach may be highly efficient in case the transformed series ${ }_{469}$ system is dominated by one or few of the composite LSF. 470

Future research efforts will aim at analyzing the application of the proposed approach con- ${ }^{471}$ sidering linearization of nonlinear stochastic processes. Another aspect to be examined is the 472 formulation of an importance sampling density function associated with the so-called static ran- ${ }_{473}$ dom variables.

\section{CRediT authorship contribution statement}

Xiukai Yuan: Conceptualization, Methodology, Software, Validation, Writing - original draft, 476 Supervision, Funding acquisition. Shaolong Liu: Methodology, Software, Writing - original draft. 477 Matthias Faes: Supervision, Writing - review \& editing, Funding acquisition. Marcos A. Valdeben- ${ }_{478}$ ito: Supervision, Writing - review \& editing, Funding acquisition. Micheal Beer: Supervision, ${ }^{479}$ Writing - review \& editing. 
The authors declare that they have no known competing financial interests or personal rela- ${ }_{482}$ tionships that could have appeared to influence the work reported in this paper.

\section{Acknowledgements}

Xiukai Yuan would like to acknowledge financial support from NSAF (Grant No. U1530122), 485 the Aeronautical Science Foundation of China (Grant No. ASFC-20170968002). Matthias Faes 486 gratefully acknowledges the financial support of the Research Foundation Flanders (FWO) under ${ }_{487}$ grant number $12 \mathrm{P} 3519 \mathrm{~N}$, as well as the Alexander von Humboldt foundation. Marcos Valdebenito 488 acknowledges the support of ANID (National Agency for Research and Development, Chile) under ${ }_{489}$ its program FONDECYT, grant number 1180271.

\section{References}

[1] K. Breitung, Asympotic Approximations for Probability Integrals, Probabilistic Engineering 492 Mechanics 4 (1989) 187-190.

[2] C. Z. Mooney, Monte carlo simulation, volume 116, Sage publications, 1997.

[3] X. Yuan, Local estimation of failure probability function by weighted approach, Probabilistic ${ }_{495}$ Engineering Mechanics 34 (2013) 1-11.

[4] S. K. Au, J. L. Beck, A new adaptive importance sampling scheme for reliability calculations, $\quad 497$ Structural Safety 21 (1999) 135-158.

[5] X. Yuan, Z. Lu, C. Zhou, Z. Yue, A novel adaptive importance sampling algorithm based on 499 markov chain and low-discrepancy sequence, Aerospace Science and Technology 29 (2013) 500 253-261. doi:https://doi.org/10.1016/j.ast.2013.03.008.

[6] R. Rackwitz, Reliability analysis - A review and some perspectives, Structural Safety 23502 (2001) 365-395.

[7] S. K. Au, J. L. Beck, Estimation of small failure probabilities in high dimensions by subset 504 simulation, Probabilistic Engineering Mechanics 16 (2001) 263-277. 
[8] M. R. Rajashekhar, B. R. Ellingwood, A new look at the response surface approach for 506 reliability analysis, Structural Safety 12 (1993) 205-220. 507

[9] B. Echard, N. Gayton, M. Lemaire, Ak-mcs: an active learning reliability method combining 508 kriging and monte carlo simulation, Structural Safety 33 (2011) 145-154. 509

[10] M. Faes, J. Sadeghi, M. Broggi, M. De Angelis, E. Patelli, M. Beer, D. Moens, On the Robust 510 Estimation of Small Failure Probabilities for Strong Nonlinear Models, ASCE-ASME Journal 511 of Risk and Uncertainty in Engineering Systems, Part B: Mechanical Engineering 5 (2019). 512

[11] U. Alibrandi, A. M. Alani, G. Ricciardi, A new sampling strategy for SVM-based response 513 surface for structural reliability analysis, Probabilistic Engineering Mechanics 41 (2015) 1-12. 514

[12] G. Blatman, B. Sudret, Adaptive sparse polynomial chaos expansion based on least angle 515 regression, Journal of Computational Physics 230 (2011) 2345-2367. 516

[13] D. Straub, R. Schneider, E. Bismut, H.-J. Kim, Reliability analysis of deteriorating structural 517 systems, Structural Safety 82 (2020) 101877.

[14] C. Andrieu-Renaud, B. Sudret, M. Lemaire, The phi2 method: a way to compute time-variant 519 reliability, Reliability Engineering \& System Safety 84 (2004) 75-86. 520

[15] Z. Hu, X. Du, Time-dependent reliability analysis with joint upcrossing rates, Structural ${ }_{521}$ and Multidisciplinary Optimization 48 (2013) 893-907. 522

[16] C. Ling, Z. Lu, K. Feng, A novel extended crossing rate method for time-dependent hybrid ${ }_{523}$ reliability analysis under random and interval inputs, Engineering Optimization (2020) 1-23. 524

[17] Z. Hu, X. Du, A sampling approach to extreme value distribution for time-dependent relia- 525 bility analysis, Journal of Mechanical Design 135 (2013). 526

[18] Z. Wang, P. Wang, A double-loop adaptive sampling approach for sensitivity-free dynamic 527 reliability analysis, Reliability Engineering \& System Safety 142 (2015) 346-356. 528

[19] Z. Hu, S. Mahadevan, A single-loop kriging surrogate modeling for time-dependent reliability ${ }_{529}$ analysis, Journal of Mechanical Design 138 (2016) 061406. 
[20] H.-M. Qian, T. Huang, H.-Z. Huang, A single-loop strategy for time-variant system reliability 531 analysis under multiple failure modes, Mechanical Systems and Signal Processing 148 (2021) 532 107159.

[21] Z. Wang, W. Chen, Time-variant reliability assessment through equivalent stochastic process 534 transformation, Reliability Engineering \& System Safety 152 (2016) 166-175. 535

[22] Z. Wang, W. Chen, Confidence-based adaptive extreme response surface for time-variant 536 reliability analysis under random excitation, Structural Safety 64 (2017) 76-86. 537

[23] C. Jiang, X. Huang, X. Han, D. Zhang, A time-variant reliability analysis method based on 538 stochastic process discretization, Journal of Mechanical Design 136 (2014). 539

[24] Z. P. Mourelatos, M. Majcher, V. Pandey, I. Baseski, Time-dependent reliability analysis 540 using the total probability theorem, Journal of Mechanical Design 137 (2015). ${ }_{541}$

[25] H.-S. Li, T. Wang, J.-Y. Yuan, H. Zhang, A sampling-based method for high-dimensional ${ }_{542}$ time-variant reliability analysis, Mechanical Systems and Signal Processing 126 (2019) 505- 543 520.

[26] W. Du, Y. Luo, Y. Wang, Time-variant reliability analysis using the parallel subset simula- ${ }_{545}$ tion, Reliability Engineering \& System Safety 182 (2019) 250-257.

[27] S. K. Au, J. L. Beck, First excursion probabilities for linear systems by very efficient impor- 547 tance sampling, Probabilistic Engineering Mechanics 16 (2001) 193-207.

[28] M. A. Misraji, M. A. Valdebenito, H. A. Jensen, C. F. Mayorga, Application of directional 549 importance sampling for estimation of first excursion probabilities of linear structural systems 550 subject to stochastic Gaussian loading, Mechanical Systems and Signal Processing 139 (2020) 551 106621. URL: https://linkinghub.elsevier.com/retrieve/pii/S0888327020300078. 552 doi:10.1016/j.ymssp.2020.106621.

[29] Z. Wang, P. Wang, A nested extreme response surface approach for time-dependent reliability- $\quad 554$ based design optimization, Journal of Mechanical Design 134 (2012). 555

[30] Y. Mori, T. Kato, Multinormal integrals by importance sampling for series system reliability, 556 $\begin{array}{ll}\text { Structural Safety } 25 \text { (2003) 363-378. } & 557\end{array}$ 
[31] E. Vanmarcke, Random Fields: Analysis and Synthesis, MIT Press, Cambridge, 1983.

[32] B. Sudret, A. Der Kiureghian, Stochastic finite element methods and reliability. A state- ${ }_{559}$ of-the-art-report, Technical Report November, Department of Civil \& Environmental Engi- 560 neering, University of California, Berkley, Institute of Structural Engineering, Mechanics and 561 Materials, 2000.

[33] S. Huang, S. Quek, K. Phoon, Convergence study of the truncated karhunen-loeve expan- 563 sion for simulation of stochastic processes, International journal for numerical methods in 564 engineering 52 (2001) 1029-1043.

[34] C.-C. Li, A. Der Kiureghian, Optimal discretization of random fields, Journal of Engineering 566 Mechanics 119 (1993) 1136-1154.

[35] W. Betz, I. Papaioannou, D. Straub, Numerical methods for the discretization of random 568 fields by means of the karhunen-loève expansion, Computer Methods in Applied Mechanics 569 and Engineering 271 (2014) 109-129.

[36] M. A. Valdebenito, H. A. Jensen, G. I. Schuëller, F. E. Caro, Reliability sensitivity estimation 571 of linear systems under stochastic excitation, Computers \& Structures 92-93 (2012) 257-268. 572 doi:10.1016/j.compstruc.2011.10.020.

[37] M. Fujita, R. Rackwitz, Updating First and Second Order Reliability Estimates by Impor- 574 tance Sampling, Structural Engineering and Earthquake Engineering, JSCE 5 (1988) 31s-37s. 575

[38] M. G. Faes, M. A. Valdebenito, D. Moens, M. Beer, Bounding the first excursion probability 576 of linear structures subjected to imprecise stochastic loading, Computers \& Structures 239577 (2020) 106320. doi:10.1016/j.compstruc.2020.106320.

[39] G. Schuëller, R. Stix, A critical appraisal of methods to determine failure probabilities, 579 Structural Safety 4 (1987) 293-309.

[40] L. Hawchar, C.-P. El Soueidy, F. Schoefs, Global kriging surrogate modeling for general 581 time-variant reliability-based design optimization problems, Structural and Multidisciplinary 582 Optimization 58 (2018) 955-968. 
[41] Y. Shi, Z. Lu, Z. Huang, L. Xu, R. He, Advanced solution strategies for time-dependent reli- ${ }_{584}$ ability based design optimization, Computer Methods in Applied Mechanics and Engineering 585 364 (2020) 112916.

[42] B. Huang, X. Du, Probabilistic uncertainty analysis by mean-value first order saddlepoint 587 approximation, Reliability Engineering \& System Safety 93 (2008) 325-336.

[43] X. Yuan, Z. Lu, Efficient approach for reliability-based optimization based on weighted 589 importance sampling approach, Reliability Engineering \& System Safety 132 (2014) 107-114. 590

[44] F. Au, Y. Cheng, L. Tham, G. Zeng, Robust design of structures using convex models, 591 Computers \& structures 81 (2003) 2611-2619.

[45] Z. Zhang, C. Jiang, X. Han, D. Hu, S. Yu, A response surface approach for structural 593 reliability analysis using evidence theory, Advances in Engineering Software 69 (2014) 37-45. 594 